\title{
Editorial
}

\section{The practice of cytopathology in Sri Lanka}

The strong presence of cytopathology in Sri Lanka is evident, by the three articles including the leading article being written on cytopathology related issues.The leading article has discussed the reporting system of thyroid cytology in Sri Lanka and compared with The Bethesda System and that of the Royal College of Pathologists, United Kingdom and highlighted the necessity of standardising the thyroid cytology reporting. The audit assesses the validity of fine needle aspiration cytology (FNAC) in the diagnosis of thyroid neoplasms, thus providing ground data for future audits. The case report bespeaks the valuable diagnostic role of FNAC.

The practice of cytopathology came to Sri Lanka in the 1980s.Although there has been no dynamic growth in this field in Sri Lanka as in the rest of the world, cytopathology has managed to establish a niche for itself that now cannot be replaced. Any potential future replacements will have to play a complementary role to cytopathology. There is no pathologist in Sri Lanka who will not be involved in practicing cytopathology as part of their daily diagnostic routine. However most of them are trained to report on cytopathology smears and continue to gain experience in the course of their practice. What is the need of the hour in the diagnostic aspect of cytopathology? The answer to this question would need assessment and consideration of the ground situation in the centres with pathology services in the country. While in the majority of centres Fine Needle Aspiration (FNA) is performed by the pathology department personnel, there are some centers which are yet to embrace this. Many a study has proven the improvement in the rate of diagnostic accuracy when FNA is performed by personnel trained in pathology/cytology(1). It being true that FNA is a training and experience dependent procedure, to be aware are the additional values of FNAC being able to assess the adequacy, nature and quality of the sample, when done by a pathology person, and this by all means may contribute to the diagnosis. Therefore the facility of onsite assessment of the aspirated material for adequacy must be made available in the centers performing FNA, which will reduce the non diagnostic rates dramatically.

It is merely going to need a microscope and training to recognize the nature of the aspirates. Efforts towards improvement in the proficiency of fine needle aspiration sampling and specimen preparation should be given priority. Performing a large number of FNA does not improve results when controlled for level of training (2). It is vital to stress the importance of obtaining an adequate sample in order for this test to be useful (2).

Most clinicians have come to terms with accepting a FNAC as a diagnostic procedure for the lumps and bumps they see in their practice. Now is the time to expand cytopathology to its fullest potential. Although clinical factors may override the cytological diagnosis in management decisions, one cannot refute the fact that FNAC diagnosis does have a strong influence on the management decisions. This is exemplified in the anatomical region of the head and neck where FNAC has undeniable value, as in diagnosing metastatic malignancy,primary tumours of the skin, lymphomas and infections such as tuberculosis (3). Not only as a tool in diagnosis, the aspirates also provide material for culture,flow cytometry,immunohistochemistry and molecular 
studies.The parameters of the accuracy of diagnosis can vary for different anatomical sites.Hence each centre must establish its own rates of diagnostic accuracy for the respective sites. This may also be a legal tool in case of diagnostic discrepancies.

Mensuration in the morphological laboratory is a subject less able to be defined than in the clinical pathology environment. Visual interpretation does not lend itself to the quality assessment and methods used in the clinical pathology laboratory (4). Nevertheless a system has to be developed to be suitably applicable to the current setup atleast in the future. In other words a viable a scheme of assessment must be in place.

There are forces influencing the evolution of cytology both locally and internationally; automation, digital imaging, molecular testing, shortage of cytopathologists and inadequate infrastructure etc (5). These collective forces may bring about the demise or ensure the viability of the practice of cytopathology, not only in Sri Lanka but also internationally. The move forward will warrant the creation of an evolved pathologist with capacity to perform beyond the hangers on of tradition and territorial domain.

\section{References}

1. Kaur G, Sivakumar S. Comparison of unsatisfactory aspirates in fine needle aspiration performed by surgical medical officers and pathologists. Journal of Cytology July 2007(8); 24: 82-4.

2. Ljung BM, et al. Diagnostic accuracy of Fine Needle Aspiration Biopsy is Determined by Physician Training in Sampling Technique. Cancer Cytopathology 2001; 93: 263-268.
(PubMed).

3. Ritu Nayar and Carlos WM Bed-rossian. Introduction. Challenges in Aspiration Cytology of the Head and Neck. Seminars in Diagnostic Pathology May 2002 (18); 2: 79-80.

4. Peter B Herdson. Forward. Quality Systems for Anatomical and Forensic Pathology Laboratories. Guidelines for implementation and Monitoring. World Health Organisation Regional Publications, Eastern Mediterranean Series 18. 1999; 5.

5. David B kaminsky. Editorial. Trans-forming Cytology. Acta Cytologica 2011; 55: 226226.

\section{Dr. CSPSosai}

Professor Neelakanthi Ratnatunga 